\title{
Unusual Pattern of Reading Errors in a Patient with Posterior Cortical Atrophy
}

\author{
Ivanna M. Pavisic ${ }^{a}$ Keir X.X. Yong ${ }^{a} \quad$ Silvia Primativo $^{b}$ \\ Sebastian J. Crutch ${ }^{a}$ Aida Suarez Gonzalez ${ }^{a}$ \\ aDementia Research Centre, Department of Neurodegenerative Diseases, Queen \\ Square Institute of Neurology, University College London (UCL), London, UK; \\ ${ }^{b}$ Department of Human Science, LUMSA University, Rome, Italy
}

\section{Keywords}

Posterior cortical atrophy · Phonological deficit · Reading · Response-stimulus interval

\begin{abstract}
Posterior cortical atrophy (PCA) is a degenerative condition characterized by a progressive deterioration of visual processing. Dyslexia constitutes an early and frequent visual symptom of the disease and previous comprehensive investigations in series of individuals have extensively documented a characteristic abundance of visual errors as the most prevalent error category in this population. Here we describe the profile of a patient with PCA, C.P., who presents an unusual prevalence of phonological, instead of purely visual, errors in his reading, in the context of an otherwise classic PCA phenotype. In keeping with the well-known PCA profile, C.P. exhibited deficits at the pre-lexical level with elements of crowding and defective early visual processing impairments but additionally showed an unusually prominent disruption of phonological processing. We also argue that our patient may have a refractory access type deficit in reading given that accuracy doubled with the introduction of a five-second responsestimulus interval. To our knowledge, no previous case of a refractory deficit affecting word reading has been reported in PCA. Our examination builds on previous knowledge about reading behaviour in PCA and describes a singular example of the rich phenotypic heterogeneity within the syndrome.




\section{Case Reports in Neurology}

\section{Introduction}

Posterior cortical atrophy (PCA) involves a progressive disintegration of visual processing skills, literacy, numeracy, gesture, and other functions that depend on parietal, occipital, and occipitotemporal cortices [1,2]. Age at onset is typically 50-65 years and PCA is typically caused by Alzheimer's disease (AD) [3].

Reading problems are amongst the earliest, most common (80-95\%), and most disabling symptoms [4]. Reading in PCA is significantly slower and less accurate than in typical AD patients and controls, with performance significantly adversely affected by increased letter spacing, size, length, and font (cursive < non-cursive) [5]. One characteristic feature of PCA reading is the high proportion of visual errors (69\% of all error responses) [5]. Reading accuracy has been associated with impairments of early visual (i.e., excessive crowding: difficulty perceiving objects in clutter), visuoperceptual, and visuospatial processes [6].

There is considerable clinical heterogeneity within the PCA spectrum, as reflected in recent consensus criteria for PCA [2]. In addition to the well-reported degradation of vision, literacy, and numeracy, PCA is characterised by progressive phonological impairment manifesting in oral language dysfunction (e.g., anomia, reduced phonemic fluency, slowed speech rate, poor non-word repetition) [7, 8]. This overlap in the linguistic profiles of PCA and logopenic progressive aphasia (LPA) [9], which are both most commonly caused by AD, emphasises the notion of a phenotypic continuum between typical and atypical manifestations of the disease. However, there have been no reported cases of PCA stressing a phonological impairment in reading.

Here we put on record a patient who, despite exhibiting a clear-cut progressive degeneration of visuoperceptual and visuospatial skills, showed single word reading behaviour that differed markedly from the majority of other reported cases by virtue of the prevalence of phonological in addition to visual errors. In this paper, "phonological" and "non-word" impairments will be used as interchangeable concepts in accordance with previously published criteria $[5,10]$.

\section{Case Report}

\section{Clinical Presentation}

C.P. is a 61-year-old right-handed man who was seen in August 2015 after a 3-year history of increasing difficulties perceiving objects and judging distances (e.g., while driving and cycling), dressing, reading, calculating, and word finding. In addition, his wife described C.P. had been tripping over his words for over a year but that his comprehension remained intact. Neuropsychological assessment at the time revealed a Mini-Mental State Examination (MMSE) of 24/30, some impairments in recognition memory for visual material (RMT) (words: 25th percentile; faces: $<5$ th percentile), and visuospatial and visuoperceptual function (i.e., Visual and Object Space Perception Battery fragmented letters: $<5$ th percentile; position discrimination: $<5$ th percentile).

No neurological or other remarkable findings arose from the medical examination. An MRI scan showed marked biparietal atrophy (Fig. 1). In November 2015, a clinical diagnosis of PCA was made. Subsequent review confirmed C.P. met research consensus criteria [2] for 
PCA at Classification Level 1 (core clinical/cognitive features), PCA-pure at Level 2 (by not additionally meeting criteria for LPA or any other neurodegenerative syndrome), and PCA-AD at Level 3 (CSF evidence consistent with AD pathology: total tau $=296$; A beta $_{1-42}=311$; ratio $=0.95$, $\mathrm{p}$-tau $=33$ ).

In 2017, at the time of the current assessment, neuropsychological assessment revealed impaired performance on testing of generalized function (MMSE $=13 / 30$ ); basic, visuospatial, and perceptual processing deficits (figure-ground discrimination $=12 / 20$; dot counting = $5 / 10$; fragmented letters $=0 / 20$; all $<5$ th percentile); mild visual crowding (letter identification with condensed flankers $=19 / 24$, spaced flankers $=24 / 24$ ); simultagnosia in the context of normal visual acuity, impaired working memory (maximum digit span: forwards $=4$; backwards $=0$; both $<5$ th percentile), and signs of limb apraxia. By contrast, episodic memory performance on the RMT words was just within normal limits $(20 / 25 ; \geq 5$ th percentile). However, he retained independence in most activities of daily living.

C.P.'s speech was fluent and grammatically correct, but with some word finding difficulties (consistent with confrontation naming to verbal description $=14 / 20 ;<1$ st percentile) and only very occasional phonological errors (i.e., "bedroom" $\rightarrow$ "beldroom"; "expect" $\rightarrow$ "exquet"; 2 phonological errors out of 207 words in spontaneous speech).

\section{Reading Assessment}

Experiments were performed between May and December 2017. During the cognitive examination, C.P.'s performance on the Schonell reading list [11] was noted to be atypical for an individual with PCA, yielding an unexpectedly high proportion of phonological (63.1\%) compared to what can be purely classified as visual errors (20.1\%). An extensive further examination of pre-lexical, lexical, and post-lexical stages of reading and an error analysis were conducted subsequently. Error classification followed Crutch and Warrington's classification [10] also used by Yong et al. [5] (Table 1). Written informed consent was provided in accordance with the guidelines established by the Declaration of Helsinki.

C.P. was assessed using five corpora of real words (total $N=480$; see Table 1). Overall, he made a significantly higher proportion of phonological or non-word than purely visual reading errors (151/309 and 79/309 errors, respectively; $\left.\chi^{2}{ }_{[1]}=35.9, p<0.001\right)$. As expected for an individual with a diagnosis of PCA, the contribution of a visual impairment to his reading performance was indeed undeniable. However, C.P.'s inability to produce a real-word response suggested an additional impairment at a phonological level, which we then evaluated further.

\section{Statistical Analysis}

Comparisons between C.P. and a group of 26 patients with PCA was performed using a modified $t$ test by Crawford and Howell [12] to identify differences between individual cases relative to a group. Reading accuracy was analysed using logistic regression and relevant lexical variables used as covariates depending on the corpus properties (i.e., Schonell [11]: length, frequency-CELEX [13], Brown and Ure words [14]: concreteness, length, frequency, Yong perceptual corpus: see [5]). For this latter, logistic regression was also covaried for perceptual variables (font size, inter-letter spacing, and case) [5]. $\chi^{2}$ test was used to compare two conditions when all other variables remained constant. 


\section{Case Reports in Neurology}

Case Rep Neurol 2019;11:157-166

DOI: $10.1159 / 00050008$

Atrophy

\section{Results}

C.P.'s reading performance was directly compared with the PCA patients reported by Yong et al. [5], the largest PCA reading case series to date $(N=26)$. Using the same error classification, C.P. made significantly more phonological errors (C.P. $=51.7 \%$; PCA group: mean $[\mathrm{SD}]=10.9$ [11.4]; $t=3.49, p$ [1-tailed] $=0.002$ ) and fewer visual errors (C.P. $=26.3 \%$; PCA group: mean $[\mathrm{SD}]=72.4 \%$ [13.3\%]; $t=3.36, p=0.002$ ) [12]. C.P.'s overall accuracy was low (C.P. $=38.5 \%$; PCA group: mean $[\mathrm{SD}]=72.3 \%$ [29.2\%]), but his error pattern seemed markedly different from even the most severely affected previous patients (e.g., Case X: accuracy = 48\%; 36\% phonological errors; $64 \%$ visual errors) (Fig. 2).

Despite a relatively low rate of visual errors compared to other PCA patients, C.P.'s reading exhibited signs of being partly influenced by pre-lexical deficits. On the Yong perceptual corpus [5], C.P. was more accurate reading words in small print $(z=-3.44, p=0.001)$, though showed no significant effect of inter-letter spacing $(z=-0.69, p=0.493)$. He was also more impaired reading a subset of cursive as compared to non-cursive print words (case) $(0 \%$ vs. $41 \%$, respectively; $\chi^{2}[1]=6.32, p=0.01$ ).

Length effects (number of letters) were only found with the Schonell reading list $(z=$ $-3.85, p<0.001$ ), potentially owing to the greater length of words (max. 14 letters vs. max. 78 letters in other corpora) increasing the impact of crowding $[5,6]$ and a reduced effect field of vision [15]. There was no evidence of a frequency effect (CELEX [13]) on reading accuracy of this corpus $(z=1.41, p=0.160)$, although higher-frequency words were read more accurately than lower-frequency words in the Yong perceptual corpus $(z=2.05, p=0.04)$. In addition to his phonological reading errors, C.P. showed no regularity effect with real words $\left(\chi^{2}{ }_{11}=0.22, p=0.639\right.$; Coltheart words [16]) and was completely unable to read non-words attempting to substitute the target for a read word (Glushko non-word corpus [17]) (see Table 1).

Although not intact, single word repetition of the McCarthy and Warrington corpus [18] was superior to reading for the same words. Words we administered using an ABBA design: repetition-reading-reading-repetition, and C.P. scored: $84 \%$ versus $29 \% ; \chi^{2}{ }_{[1]}=56.91, p<$ 0.001 . C.P. was able to repeat 57 words that he was, however, not able to read (82.6\%). Additionally, cliché repetition, which is more reliant on phonological processing and less on semantic processing than sentences [18], was markedly impaired (clichés $=30 \%$; sentences $=$ $80 \% ; \chi^{2}[1]=5.05, p=0.03$; "Take it with a pinch of salt" $\rightarrow$ "Pake it with a sprint of salt."

With the aim of evaluating the atypicality of C.P.'s reading further, 72 words of varying length from the Brown and Ure corpus [14] (matched for lexical variables, i.e., CELEX frequency, concreteness) were presented at two response-stimulus intervals (RSI) (normal pace between responses, 5-second delay between responses) to evaluate the presence of any temporal effects. The 5-second RSI doubled reading accuracy both for concrete and abstract words $(z=2.08, p=0.037$ and $z=2.06, p=0.039$, respectively) (Table 2). Interestingly, a greater delay between responses increased C.P.'s proportion of real-word responses and decreased his non-word responses (Table 2).

Given the diagnosis of PCA and the undeniable visual impairment, it is expected that phonological errors would be visually related to the target. To quantify this, we considered these phonological errors to be visually related to the target if at least $50 \%$ of the letters in the nonword output were maintained. Based on these criteria, over half of non-word errors were 


\section{Case Reports in Neurology}

Case Rep Neurol 2019;11:157-166

DOI: $10.1159 / 00050008$

2019 The Author(s). Published by S. Karger AG, Basel www.karger.com/crn

Pavisic et al.: Unusual Pattern of Reading Errors in a Patient with Posterior Cortical Atrophy

visually related to the target (overall: 70/126, 55.6\%; Schonell reading list: 26/41, 63.1\%; 192 Yong perceptual corpus: 29/61, 47.5\%; Coltheart words: 1/3, 33.3\%; Brown and Ure words: $12 / 18,66.7 \%$, and Glushko non-word corpus: $2 / 3,66.7 \%$ ).

\section{Discussion}

Despite fulfilling criteria for PCA and not LPA, C.P. showed a significant phonological deficit in addition to his visual processing impairments. These errors were manifested primarily in phonological/non-word reading errors and were beyond the extent of those reported in previous PCA patients.

\section{Early Visual/Pre-Lexical Processing}

C.P. was less accurate reading words of large font size than small. This has been referred to as an inverse size effect, previously attributed to a shrinkage of the effective field of vision, and is one of the possible reasons for C.P.'s poor performances in accuracy of reading long words in the Schonell reading list, for instance. The Schonell comprises words up to 14 letters in length, with word length confounded by the corpus being unbalanced in frequency for words of different lengths. Although frequency was a covariate in the model, this effect could arise for several reasons: the increased number of letters in longer words may operate as multiple flankers, elevating the strength of the crowding effect [19] and long words may also behave as perceptually larger stimuli, which might extend further into the periphery, beyond the effective visual field [5]. Indeed, C.P. exhibited excessive visual crowding based on particular difficulty identifying letters with condensed relative to spaced flankers.

C.P. had marked difficulties reading cursive script and was unable to read any words presented in such font, consistent with previous series of PCA patients [5]. No statistically significant effect of inter-letter spacing was observed, although there seemed to be a trend towards a detrimental effect of inter-letter spacing. There is certain controversy in the literature around the benefit of inter-letter spacing in reading in PCA $[5,6,10]$, which is likely due to differences in the threshold for inter-letter spacing. While a certain degree has proved beneficial, wider values might destroy the word form and therefore disrupt reading. Such effects might be indicative of a disruption in the word form processing system, unable to accommodate word processing under perceptually unfamiliar and/or complex presentations.

Taken together, these results summarize evidence in support of our patient having visual crowding, disruption in the word form processing system, and restriction in the effective visual field.

\section{Central Loci}

C.P. was entirely unable to read non-words aloud, a task aiming to test the integrity of phonological processing and previously reported as impaired in PCA $[8,10]$. Moreover, as explained by Mendez and colleagues [8], the need to revert to serial analysis for non-words, as opposed to real words, generates a greater predisposition to visual word errors and this was also the case for C.P. [8]. Reading non-words requires selective attention and serial analysis, which is a possible explanation as to why C.P. and other PCA patients make a disproportionate 
number of visual errors, many of which lead to lexicalizations. Nonetheless, this does not exclude the possibility of an underlying phonological deficit [8].

In addition, his reading did not show a regularity effect, in line with Mendez and colleagues [8]. These results provide evidence supporting the notion of a disruption at the level of the phonological process and suggest an additional defect either at the level of the word form units or their activation process.

No frequency effects were observed when reading the Schonell reading list. The Schonell is a test originally conceived to look at reading in different ages, for vocabulary purposes, and an average adult is expected to perform at ceiling on it and is therefore probably not the most robust to look at frequency effects. The Yong Perceptual corpus did, however, reveal a frequency effect. Given that this corpus was designed balancing this lexical variable, frequency will be briefly addressed. A possible explanation for this effect is a disruption of the sub-lexi$\mathrm{cal} /$ grapheme-phoneme conversion route (given that low-frequency words are likely to recruit this route).

\section{Phonological Errors and Language}

C.P.'s imperfect repetition but greater deficiency in reading suggests a post-lexical language component that could in fact be contributing to poor reading performance. His lower accuracy in repeating clichés compared to sentences further supports the presence of a phonological deficit. Sentences retain semantic meaning, and this makes it easier to rely on semantics to produce a verbal response, while clichés may be meaningless and therefore force repetition to relay on mere phonology [18].

Additionally, C.P.'s improvement in reading with the addition of the 5-second RSI might suggest a refractory effect present in reading. Similar effects have been reported previously in patients with non-fluent aphasia and attributed to a deficit at a phonology level [20]. Although accuracy levels remained relatively low with the 5-second RSI, an analogous explanation may be possible as the accuracy doubled.

We suggest this phonological impairment cannot be accounted for by a general degradation or progression of the disease. This impairment appeared as an early symptom around the time of his diagnosis and C.P. retained autonomy in most activities of daily living at the time of the assessment. His general function (MMSE) was within one standard deviation from PCA group study [5], and his reading error patterns were markedly different (greater percentage of phonological errors in addition to visual errors) even from the most severely affected previous PCA patients (participant 1: MMSE = 10; participant 2: MMSE = 13) (Fig. 2) [5].

\section{Summary}

C.P.'s reading shares certain similarities with previously reported PCA cases at a pre-lexical deficit, demonstrating excessive visual crowding and an inverse size effect. However, different to other PCA cases, C.P. also displays an unusual disruption in his phonological processing predominant (although not entirely selective) to reading that may well have a refractory component to it.

Naturally, this does not represent an uncontaminated example of a phonological deficit in reading. However, in dementia syndromes, very few phenomena are pure, and lesions are 
rarely (if ever) focal. For this reason, we believe the current case is a remarkable example of the clinical variability of the condition, promoting understanding of the susceptibility of reading not only to peripheral, but also phonological deficits in this syndrome.

\section{Acknowledgement}

We would like to thank C.P. and his wife for generously providing us with their time and Professor Elizabeth Warrington for her guidance with the work.

\section{Statement of Ethics}

Written informed consent was provided in accordance with the guidelines established by the Declaration of Helsinki.

\section{Disclosure Statement}

The authors have no conflicts of interest to declare.

\section{Funding Sources}

This work was undertaken at UCLH/UCL, which received a proportion of funding from the Department of Health's NIHR Biomedical Research Centre funding scheme. The Dementia Research Centre is supported by Alzheimer's Research UK, Brain Research Trust, and The Wolfson Foundation. This work was supported by the NIHR Queen Square Dementia Biomedical Research Unit and by an Alzheimer's Research UK Senior Research Fellowship and ESRC/NIHR (ES/K006711/1) and EPSRC (EP/M006093/1) grants to S.J.C. K.X.X.Y. is funded by Alzheimer's Society.

\section{Author Contributions}

I.M.P.: Substantial contributions to the conception or design of the work, acquisition, analysis and interpretation of data for the work; drafting the work; final approval for the version to be published and agreement to be accountable for all aspects of the work in ensuring that questions related to the accuracy or integrity of any parts of the work are appropriately investigated and resolved. K.X.X.Y.: Substantial contributions to the acquisition of the work; revising the work critically for important intellectual content; final approval for the version to be published and agreement to be accountable for all aspects of the work in ensuring that questions related to the accuracy or integrity of any parts of the work are appropriately investigated and resolved. S.P.: Substantial contributions to the interpretation of data for the work; revising the work critically for important intellectual content; final approval for the 
version to be published and agreement to be accountable for all aspects of the work in ensuring that questions related to the accuracy or integrity of any parts of the work are appropriately investigated and resolved. S.J.C.: Substantial contributions to the interpretation of data for the work; revising the work critically for important intellectual content; final approval for the version to be published and agreement to be accountable for all aspects of the work in ensuring that questions related to the accuracy or integrity of any parts of the work are appropriately investigated and resolved. A.S.G.: Substantial contributions to the conception and interpretation of data for the work; drafting the work and revising it critically for important intellectual content; final approval for the version to be published and agreement to be accountable for all aspects of the work in ensuring that questions related to the accuracy or integrity of any parts of the work are appropriately investigated and resolved.

\section{References}

1 Benson DF, Davis RJ, Snyder BD. Posterior cortical atrophy. Arch Neurol. 1988 Jul;45(7):789-93.

2 Crutch SJ, Schott JM, Rabinovici GD, Murray M, Snowden JS, van der Flier WM, et al.; Alzheimer's Association ISTAART Atypical Alzheimer's Disease and Associated Syndromes Professional Interest Area. Consensus classification of posterior cortical atrophy. Alzheimers Dement. 2017 Aug;13(8):870-84.

3 Tang-Wai DF, Graff-Radford NR, Boeve BF, Dickson DW, Parisi JE, Crook R, et al. Clinical, genetic, and neuropathologic characteristics of posterior cortical atrophy. Neurology. 2004 Oct;63(7):1168-74.

4 McMonagle P, Deering F, Berliner Y, Kertesz A. The cognitive profile of posterior cortical atrophy. Neurology. 2006 Feb;66(3):331-8.

5 Yong KX, Shakespeare TJ, Cash D, Henley SM, Warren JD, Crutch SJ. (Con)text-specific effects of visual dysfunction on reading in posterior cortical atrophy. Cortex. 2014 Aug;57:92-106.

6 Yong KX, Shakespeare TJ, Cash D, Henley SM, Nicholas JM, Ridgway GR, et al. Prominent effects and neural correlates of visual crowding in a neurodegenerative disease population. Brain. 2014 Dec;137(Pt 12):328499.

7 Crutch SJ, Lehmann M, Warren JD, Rohrer JD. The language profile of posterior cortical atrophy. J Neurol Neurosurg Psychiatry. 2013 Apr;84(4):460-6.

8 Mendez MF, Shapira JS, Clark DG. "Apperceptive” alexia in posterior cortical atrophy. Cortex. 2007 Feb;43(2):264-70.

9 Gorno-Tempini ML, Brambati SM, Ginex V, Ogar J, Dronkers NF, Marcone A, et al. The logopenic/phonological variant of primary progressive aphasia. Neurology. 2008 Oct;71(16):1227-34.

10 Crutch SJ, Warrington EK. The relationship between visual crowding and letter confusability: towards an understanding of dyslexia in posterior cortical atrophy. Cogn Neuropsychol. 2009 Jul;26(5):471-98.

11 Schonell FJ. The Psychology and Teaching of Reading. Oliver and Boyd; 1971.

12 Crawford JR, Howell DC. Comparing an Individual's Test Score Against Norms Derived from Small Samples. Clin Neuropsychol. 1998 Nov;12(4):482-6.

13 Baayen RH, Piepenbrock R, van Rijn H. The CELEX Lexical Database (CD-ROM). Linguistic Data Consortium, University of Pennsylvania; 1993.

14 Brown WP, Ure DM. Five rated characteristics of 650 word association stimuli. Br J Psychol. 1969 May;60(2):233-49.

15 Russell C, Malhotra P, Husain M. Attention modulates the visual field in healthy observers and parietal patients. Neuroreport. 2004 Oct;15(14):2189-93.

16 Coltheart M, Besner D, Jonasson JT, Davelaar E. Phonological encoding in the lexical decision task. Q J Exp Psychol. 1979 Aug;31(3):489-507.

17 Glushko RJ. The organization and activation of orthographic knowledge in reading aloud. J Exp Psychol Hum Percept Perform. 1979;5(4):674-91.

18 McCarthy R, Warrington EK. A two-route model of speech production. Evidence from aphasia. Brain. 1984 Jun;107(Pt 2):463-85.

19 Põder E, Wagemans J. Crowding with conjunctions of simple features. J Vis. 2007 Nov 20;7(2):23.1-12.

20 Crutch SJ, Warrington EK. Refractory dyslexia: evidence of multiple task-specific phonological output stores. Brain. 2001 Aug;124(Pt 8):1533-43. 


\section{Case Reports in Neurology}

\section{Case Rep Neurol 2019;11:157-166}

DOI: $10.1159 / 00050008$

(C) 2019 The Author(s). Published by S. Karger AG, Basel www.karger.com/crn

Pavisic et al.: Unusual Pattern of Reading Errors in a Patient with Posterior Cortical Atrophy
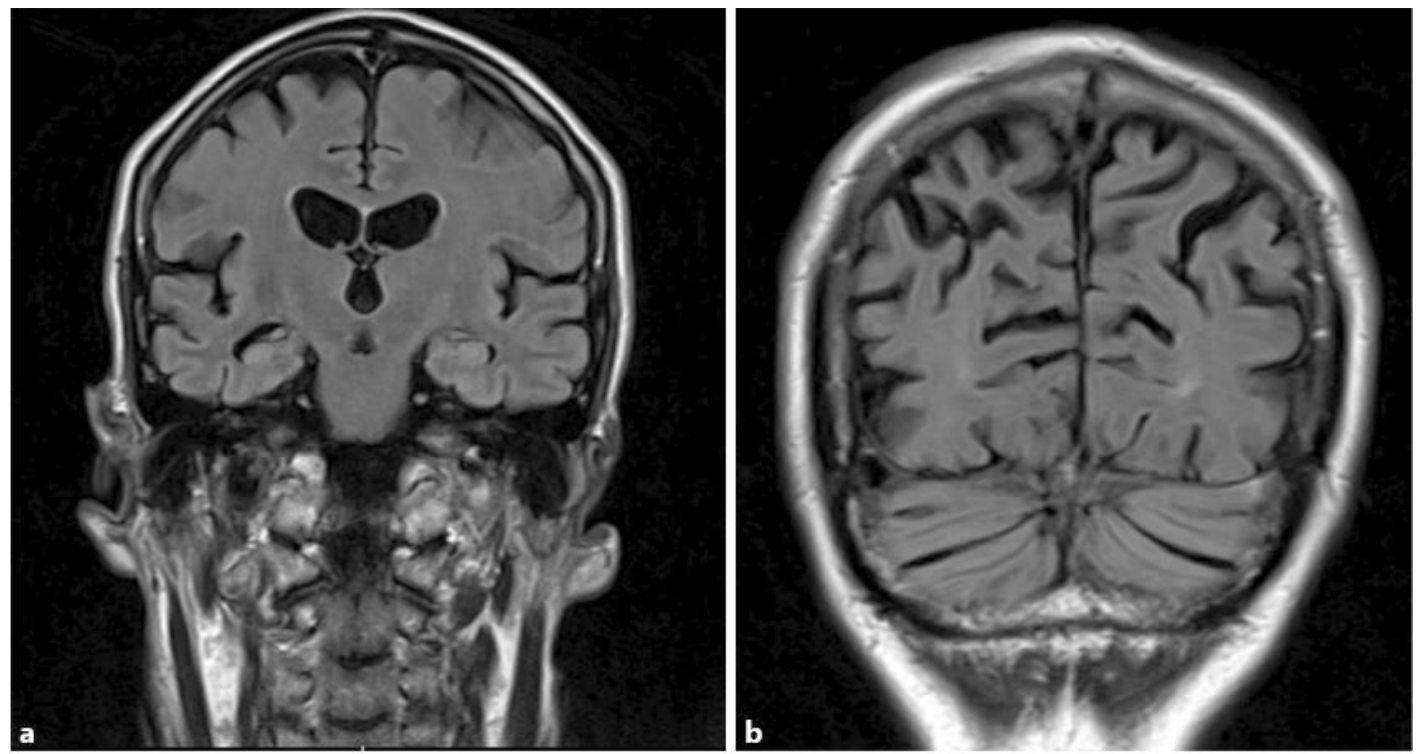

Fig. 1. a, b Patient C.P.: MRI coronal view showing biparietal atrophy with the right side more atrophied than the left.

\begin{tabular}{|c|c|c|c|c|c|c|}
\hline Target word & $\begin{array}{c}\text { Participant } 1 \\
\text { (MMSE=10; } \\
\text { age=53) }\end{array}$ & $\begin{array}{l}\text { Error } \\
\text { type }\end{array}$ & $\begin{array}{c}\text { Participant } 2 \\
\text { (MMSE=13; } \\
\text { age }=61 \text { ) }\end{array}$ & $\begin{array}{l}\text { Error } \\
\text { type }\end{array}$ & CP (MMSE $=13 ;$ age $=61$ ) & $\begin{array}{l}\text { Error } \\
\text { type }\end{array}$ \\
\hline SER VANT & & & raspberry & v & stree stree & P \\
\hline VAN & & & ant & V & vai vernin & $P$ \\
\hline G R A V Y & green & $M$ & gill & M & gra graing grainging & $P$ \\
\hline FREEDOM & from & V & & & fai se seminen free threeden & $\mathrm{P}$ \\
\hline DOUGH & door & M & brush & M & da doubt da & $P$ \\
\hline DREAMER & dream & $\mathrm{D} / \mathrm{V}$ & vine & $M$ & dree dreening & P \\
\hline JANITOR & & & teacher & $M$ & trainor trainor & V \\
\hline sap & & & & & sss say sap sap & \\
\hline gallery & & & & & ehh gah gaery ga gae & $\mathbf{P}$ \\
\hline pound & & & & & gund, ground, and, aone & $P$ \\
\hline e If & & & & & $\mathrm{t}$ trelf & $\mathrm{P}$ \\
\hline
\end{tabular}

Fig. 2. Examples of C.P.'s responses to the Yong perceptual corpus compared to two other PCA patients with similar MMSEs. When more than one response was provided, the last response was considered. Cells were left blank when the correct response was provided as a first attempt. Type of error: P, non-word/phonological; V, visual; O, omission; M, miscellaneous; D/V, derivational/visual error (in accordance with [5], [10]). 
Pavisic et al.: Unusual Pattern of Reading Errors in a Patient with Posterior Cortical Atrophy

Table 1. Number and percentage of errors made in each of the reading corpora (listed in order of size of corpus)

\begin{tabular}{|c|c|c|c|c|c|c|c|}
\hline Type of error & $\begin{array}{l}\text { Yong } \\
\text { perceptual } \\
\text { corpus [5] } \\
(n=192)\end{array}$ & $\begin{array}{l}\text { Schonell } \\
\text { reading list } \\
{[11]} \\
(n=100)\end{array}$ & $\begin{array}{l}\text { McCarthy and } \\
\text { Warrington } \\
\text { corpus }^{1}[18] \\
(n=96)\end{array}$ & $\begin{array}{l}\text { Brown and } \\
\text { Ure words [13] } \\
(n=72)\end{array}$ & $\begin{array}{l}\text { Coltheart } \\
\text { regular/ } \\
\text { irregular } \\
\text { words [16] } \\
(n=20)\end{array}$ & $\begin{array}{l}\text { Glushko } \\
\text { non-word } \\
\text { corpus }^{2}[17] \\
(n=10)\end{array}$ & $\begin{array}{l}\text { Total real-word } \\
\text { errors } \\
(n=480)\end{array}$ \\
\hline Visual & $31(26.3 \%)$ & $13(20.1 \%)$ & $14(20.6 \%)$ & $16(35.5 \%)$ & $5(25 \%)$ & $6(60.0 \%)$ & $79(25.6 \%)$ \\
\hline Phonological/non-word & $61(51.7 \%)$ & $41(63.1 \%)$ & $28(41.2 \%)$ & $18(40.0 \%)$ & $3(15 \%)$ & $3(30.0 \%)$ & $151(48.9 \%)$ \\
\hline Visual/semantic & $0(0 \%)$ & $0(0 \%)$ & $0(0 \%)$ & $0(0 \%)$ & $0(0 \%)$ & $0(0 \%)$ & $0(0 \%)$ \\
\hline Semantic & $0(0 \%)$ & $0(0 \%)$ & $2(2.9 \%)$ & $0(0 \%)$ & $1(5 \%)$ & $0(0 \%)$ & $3(1.0 \%)$ \\
\hline Morphological & $0(0 \%)$ & $6(9.2 \%)$ & $2(2.9 \%)$ & $1(2.2 \%)$ & $1(5 \%)$ & $0(0 \%)$ & $10(3.2 \%)$ \\
\hline Circumlocution & $0(0 \%)$ & $1(1.5 \%)$ & $0(0 \%)$ & $0(0 \%)$ & $0(0 \%)$ & $0(0 \%)$ & $1(0.3 \%)$ \\
\hline Miscellaneous & $22(18.6 \%)$ & $3(4.6 \%)$ & $10(14.7 \%)$ & $7(15.6 \%)$ & $0(0 \%)$ & $1(10.0 \%)$ & $42(13.6 \%)$ \\
\hline Omission & $4(3.4 \%)$ & $1(1.5 \%)$ & $12(17.7 \%)$ & $3(6.7 \%)$ & $3(15 \%)$ & $0(0 \%)$ & $23(7.4 \%)$ \\
\hline Total errors & $118(61.5 \%)$ & $65(65.0 \%)$ & $68(70.8 \%)$ & $45(62.5 \%)$ & $13(65 \%)$ & $10(100 \%)$ & $309(64.4 \%)$ \\
\hline
\end{tabular}

Error classification followed criteria used by Crutch and Warrington [10] for visual errors (real-word errors in which at least 50 percent of the letters are maintained [e.g., quarrel $\rightarrow$ squirrel]), phonological/non-word errors (addition, deletion, or substitution of one or more target phonemes yielding a non-word error [e.g., retreat $\rightarrow$ retear]), and other error types. ${ }^{1}$ The McCarthy and Warrington corpus was used not only for reading but also to compare repetition. ${ }^{2}$ In the Glushko non-word corpus, unlike the other corpuses, the stimuli are non-words.

Table 2. Number and percentage of errors in the Brown and Ure words [13]: errors are divided in terms of response-stimulus intervals (RSI) and concreteness

\begin{tabular}{llllll}
\hline \multirow{2}{*}{ Type of error } & \multicolumn{2}{l}{ Concrete words } & & \multicolumn{2}{l}{ Abstract words } \\
\cline { 2 - 3 } \cline { 5 - 6 } & normal pace & 5-second RSI & & normal pace & 5 -second RSI \\
\hline Visual & $2(16.7 \%)$ & $4(57.1 \%)$ & & $4(26.7 \%)$ & $6(50.0 \%)$ \\
Phonological/non-word & $6(50.0 \%)$ & $2(28.6 \%)$ & & $7(46.7 \%)$ & $3(25.0 \%)$ \\
Visual/semantic & $0(0 \%)$ & $0(0 \%)$ & & $0(0 \%)$ & $1(8.3 \%)$ \\
Semantic & $0(0 \%)$ & $0(0 \%)$ & & $0(0 \%)$ & $0(0 \%)$ \\
Morphological & $0(0 \%)$ & $0(0 \%)$ & & $1(6.6 \%)$ & $0(0 \%)$ \\
Circumlocution & $0(0 \%)$ & $0(0 \%)$ & & $0(0 \%)$ & $0(0 \%)$ \\
Miscellaneous & $1(8.3 \%)$ & $1(14.3 \%)$ & & $3(20.0 \%)$ & $2(16.7 \%)$ \\
Omission & $3(25.0 \%)$ & $0(0 \%)$ & & $0(0 \%)$ & $0(0 \%)$ \\
\hline Errors & $12(66.6 \%)$ & $7(38.9 \%)$ & $15(83.3 \%)$ & $12(66.6 \%)$ \\
\hline Total correct & $6(33.4 \%)$ & $11(61.1 \%)$ & $3(16.7 \%)$ & $6(33.4 \%)$ \\
\hline Total errors & & $19(52.8 \%)$ & & $27(75.0 \%)$ \\
\hline
\end{tabular}

